\section{Jerk-Limited Time-Optimal Reference Trajectory Generation for Robot Actuators}

\author{
Byeong-Ju Park ${ }^{1}$, Hong-Jun Lee ${ }^{2}$, Kwang-Kyo Oh ${ }^{3}$, and Chae-Joo Moon ${ }^{1}$ \\ ${ }^{1}$ Department of Electrical Engineering, Mokpo National University, Mokpo, Korea \\ ${ }^{2}$ School of Mechanical Engineering, Gwangju Institute of Science and Technology, Gwangju, Korea \\ ${ }^{3}$ Automotive Components and Materials R\&D Group, Korea Institute of Industrial Technology, Gwangju, \\ Korea
}

\section{]jfis}

\begin{abstract}
Smooth trajectory generation is crucial for high performance control of actuators in industrial robots. In this article, we implement a simple jerk-limited time-optimal point-to-point reference trajectories for position control of electric motor based actuators. First, we briefly review the jerk-limited reference trajectory generation schemes. Then we simplify an existing result under the assumption that the initial and the final speeds of each reference trajectory are zero. Experimental results are provided to show the validity of the simplified algorithm.
\end{abstract}

Keywords: Robots, Jerk-limited, Time-optimal trajectory, Polynomial interpolation

\section{Introduction}

Industrial robots require smooth reference trajectories to achieve high performance in motion control of their actuators [1]. In the motion control of electric motor based actuators, a reference trajectory that is not sufficiently smooth might cause undesirable phenomena such as degradation of control performance, mechanical vibration, over-current of drives, etc. The necessity for such smooth reference trajectories arise from the physical constraints imposed on electric motors and drives [2]. First, the acceleration trajectory of an actuator is essentially continuous, which implies the necessity for the continuity of the reference acceleration trajectory. To see the continuity of the acceleration trajectory, recall that the acceleration of the actuator is proportional to the stator current. Since the stator current is continuous, i.e., it cannot change abruptly because of the stator inductance, it is clear that the acceleration

Received: Nov. 202017

Revised : Dec. 17, 2017

Accepted: Dec. 18, 2017

Correspondence to: Chae-Joo Moon

(cjmoon@mokpo.ac.kr)

@The Korean Institute of Intelligent Systems

cCThis is an Open Access article distributed under the terms of the Creative Commons Attribution Non-Commercial License (http://creativecommons.org/licenses/ by-nc/3.0// which permits unrestricted noncommercial use, distribution, and reproduction in any medium, provided the original work is properly cited. trajectory is always continuous. This constraint can be expressed as the boundedness of jerk, which is the instantaneous change rate of the acceleration. Second, the acceleration is bounded. This is due to the absolute rating of the electric motor and drive consisting of the actuator. Third, the speed of the actuator is bounded by the properties of the electric motor and drive. This is related to the electro-motive force generated by the electric motor and the DC link voltage of the drive. Thus, when planning the reference position trajectories, such physical constraints needs to be considered for the high performance motion control of the actuator.

Considering such physical constraints, trajectory generation schemes have been proposed [1, 3-6]. Among the existing schemes, polynomial interpolation based schemes have attracted research interests [3, 4]. Though smooth trajectories can be obtained based on high-order polynomial interpolation methods, they cause heavy computational load, which makes it 
difficult to implement.

To overcome the problem of the heavy computation load, the authors of [3] proposed a smooth speed reference generation algorithm using a fifth-order polynomial interpolation. Based on the result in [3], jerk-limited time-optimal reference speed trajectories can be obtained. The authors of [4] presented a jerklimited time-optimal reference position trajectories. Though the algorithm presented in [4] is efficient and easy to implement, its computational load is still too heavy to be implemented in cost-effective microcontrollers.

Based on the above observations, we implement a simple jerk-limited time-optimal reference speed trajectories. To make the generation algorithm simple enough to be implemented in a cost-effective microcontroller, we assume that the acceleration and speed values at the initial and final instances are zero. Due to this assumption, reference trajectories become symmetric, which allows us to reduce the computation load. This simplified algorithm is implemented in a cost-effective microcontroller.

The remainder of this article is organized as follows. First, we briefly review the polynomial based jerk-limited reference trajectory generation in Section 2. In Section 3, we present the algorithm for the generation of jerk-limited time-optimal reference position trajectory under the assumption that the acceleration and speed values at the initial and the final instances are all zero. Simulation and experiment results are provided in Sections 4 and 5. We conclude the paper in Section 6.

\section{Piecewise Polynomial based Jerk-Limited Time- Optimal Trajectories}

When generating reference speed trajectories, we need to consider the following conditions and constraints:

$$
\begin{aligned}
& p(0)=p_{0}, p(T)=p_{f}, \\
& \dot{p}(0)=v_{0}, \dot{p}(T)=v_{f}, \\
& \ddot{p}(0)=a_{0}, \ddot{p}(T)=a_{f},
\end{aligned}
$$

where $p$ denotes the position (or angle) of the actuator, and

$$
\begin{aligned}
& -V \leq \dot{p}(t) \leq V, \\
& -D \leq \ddot{p}(t) \leq A, \\
& -J \leq \dddot{p}(t) \leq J,
\end{aligned}
$$

where $V$ denotes the maximum speed, $A$ and $D$ denote the maximum acceleration and deceleration, respectively, and $J$ denotes the maximum jerk. The conditions in (1a) specify the position, speed, and acceleration at the initial and the final instances. The constraints in $2 \mathrm{a}$ ) show the physical constraints of the actuator.

A generation algorithm for jerk-limited time-optimal reference trajectories was presented in [4] assuming that $a_{0}=a_{f}=0$. By the jerk-limited time-optimal trajectory for given (1a) and 2a), we mean the trajectory $p(t)$ that minimizes the traveling period $T$ while satisfying the boundary conditions and physical constraints.

Based on the algorithm in [4], the jerk-limited time-optimal reference position trajectories for given (1a) and (2a) can be found as follows:

$$
p(t)= \begin{cases}p_{0}+v_{0} \tau_{1}+J \tau_{1}^{3} / 6, & t_{0} \leq t<t_{1}, \\ p_{1}+v_{1} \tau_{2}+\frac{1}{2} A \tau_{2}^{2}, & t_{1} \leq t<t_{2}, \\ p_{2}+v_{2} \tau_{3}+\frac{1}{2} A \tau_{3}^{2}-J \tau_{3}^{3} / 6, & t_{2} \leq t<t_{3}, \\ p_{3}+v_{3} \tau_{4}, & t_{3} \leq t<t_{4}, \\ p_{4}+v_{4} \tau_{5}-J \tau_{5}^{3} / 6, & t_{4} \leq t<t_{5}, \\ p_{5}+v_{5} \tau_{6}-\frac{1}{2} D \tau_{6}^{2}, & t_{5} \leq t<t_{6}, \\ p_{6}+v_{6} \tau_{7}-\frac{1}{2} D \tau_{7}^{2}+J \tau_{7}^{3} / 6, & t_{6} \leq t<t_{7},\end{cases}
$$

where $\tau_{k}=t-t_{k-1}$ and

$$
\begin{aligned}
& p_{1}=p_{0}+v_{0} \tau_{1}+J \tau_{1}^{3} / 6, \\
& p_{2}=p_{1}+v_{1} \tau_{2}+\frac{1}{2} A \tau_{2}^{2}, \\
& p_{3}=p_{2}+v_{2} \tau_{3}+\frac{1}{2} A \tau_{3}^{2}-J \tau_{3}^{3} / 6, \\
& p_{4}=p_{3}+v_{3} \tau_{4} \\
& p_{5}=p_{4}+v_{4} \tau_{5}-J \tau_{5}^{3} / 6 \\
& p_{6}=p_{5}+v_{5} \tau_{6}-\frac{1}{2} D \tau_{6}^{2} \\
& v_{1}=v_{0}+\frac{1}{2} J \tau_{1}^{2} \\
& v_{2}=v_{1}+A \tau_{2} \\
& v_{3}=v_{2}+A \tau_{3}-\frac{1}{2} J \tau_{3}^{2} \\
& v_{4}=v_{3} \\
& v_{5}=v_{4}-\frac{1}{2} J \tau_{5}^{2} \\
& v_{6}=v_{5}-D \tau_{6} .
\end{aligned}
$$

It should be noted here that the generation algorithm in [4] requires considerable amount of computation to find the time parameters, $t_{1}, \ldots, t_{7}$, though the reference position trajectories 
are given by (3).

\section{Simplified Version of Jerk-Limited Time- Optimal Trajectory Generation Algorithm}

Since the computational load required by the algorithm in [4] is not ignorable when the algorithm is implemented in a costeffective microcontroller, it is desirable to have a simplified version of the algorithm. To this end, we additionally assume that $v_{0}=v_{f}=0$, which allows us to simplify the algorithm based on the symmetry of the reference trajectories. Though the simplified version of the algorithm is less general, it is expected that the algorithm can be used for low-cost robotic systems.

The conditions for the generation of jerk-limited time-optimal reference position trajectories are summarized as follows:

$$
\begin{aligned}
& p(0)=p_{0}, p(T)=p_{f}, \\
& \dot{p}(0)=0, \dot{p}(T)=0, \\
& \ddot{p}(0)=0, \ddot{p}(T)=0 .
\end{aligned}
$$

The physical constraints to be considered are as follows:

$$
\begin{aligned}
& -V \leq \dot{p}(t) \leq V, \\
& -A \leq \ddot{p}(t) \leq A,
\end{aligned}
$$

and

$$
\dddot{p}(t)= \begin{cases}J, & a(t)>0, \\ -J, & a(t)<0, \\ 0, & a(t)=0 .\end{cases}
$$

Further we assume that the acceleration rate and the deceleration rate are identical for simplicity, i.e., $A=D$.

Depending on whether the speed and the acceleration values are limited in (5), we can consider the following three cases:

- CASE I: Both of the reference speed and acceleration reach their maximum values;

- CASE II: The reference acceleration reaches its maximum value while the reference speed does not;

- CASE III: Both of the reference speed and acceleration do not reach their maximum values.

We need not consider the case in which the reference speed reaches its maximum value while the reference acceleration does not. This case does not make sure the time-optimality of the reference trajectory. In the following, we present the jerk-limited time-optimal trajectory for each case.

For brevity, we define $T_{k}=t_{k}-t_{k-1}$. Due to the conditions in and the assumption that $A=D$, the reference position trajectory has point symmetry with the center at $\left(\frac{T}{2}, \frac{p_{0}+p_{f}}{2}\right)$. Based on this fact, we obtain the following conditions:

$$
\begin{aligned}
& T_{1}=T_{7}, \\
& T_{2}=T_{6}, \\
& T_{3}=T_{5} .
\end{aligned}
$$

Due to the assumption that $A=D$, we obtain $T_{1}=T_{3}=T_{5}=T_{7}$. Further the traveling period can be divided into the acceleration, constant speed, and deceleration periods in general. For brevity, we denote the periods by $X_{1}, X_{2}$, and $X_{3}$, respectively. Definitions for the periods are given as follows:

$$
\begin{aligned}
& X_{1}=T_{1}+T_{2}+T_{3}, \\
& X_{2}=T_{4}, \\
& X_{3}=T_{5}+T_{6}+T_{6} .
\end{aligned}
$$

By the point symmetry, the traveling distance $L$ by the reference position trajectory is given as

$$
L=\frac{1}{2} v_{p} X_{1}+v_{p} X_{2}+\frac{1}{2} v_{p} X_{3},
$$

where $v_{p}$ is the peak speed during the traveling. The peak speed $v_{p}$ is given as

$$
v_{p}=\frac{1}{2} A T_{1}+A T_{2}+\frac{1}{2} A T_{3},
$$

which will be clear in below. Note that the traveling distance $L$ needs to be the given distance $P=p(T)-p(0)$.

\subsection{Reference Trajectory for CASE I}

We first consider the case that both of the reference speed and acceleration reach their maximum values. That is the case that $v(t)$ and $a(t)$ have $V$ and $A$ at some instances. A typical jerklimited time-optimal reference position trajectory is shown in Figure 1. As shown in Figure 1, the reference acceleration reaches its maximum value $A$ in $T_{2}$ interval and the reference speed reaches its maximum value $V$ in $T_{4}$.

Since the reference speed reaches its maximum value $V$ in $T_{4}$, the peak speed $v_{p}$ of the trajectory can be obtained as

$$
v_{p}=V .
$$




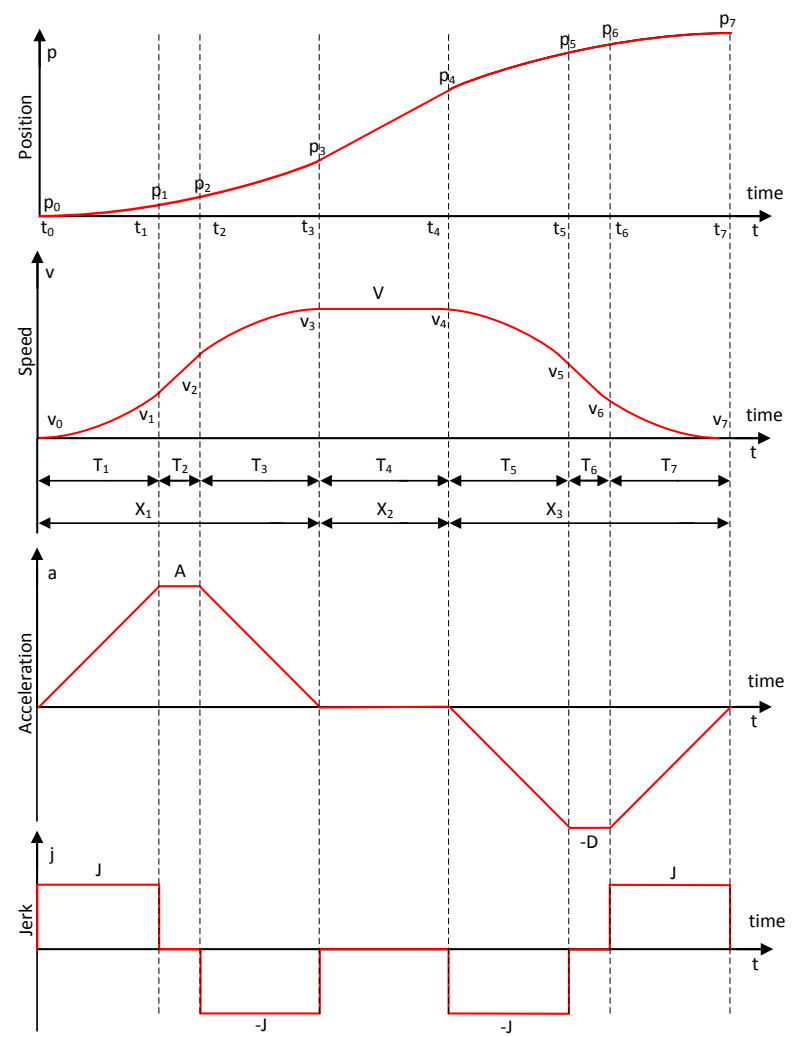

Figure 1. An Example of reference trajectory for CASE I.

Further the acceleration period $T_{1}$ is obtained as

$$
T_{1}=\frac{A}{J}
$$

because the reference acceleration reaches its maximum value $A$ and the jerk needs to be its maximum value $J$ during the acceleration period to make sure the time-optimality. From the condition that the reference speed at the instance $t=t_{3}$ is $V$, we have

$$
\frac{1}{2} A T_{1}+A T_{2}+\frac{1}{2} A T_{3}=V
$$

which leads us to

$$
T_{2}=\frac{V-A T_{1}}{A}
$$

To make sure that $L=P$, we have

$$
\frac{1}{2} V X_{1}+V X_{2}+\frac{1}{2} V X_{3}=P
$$

which allows us to obtain

$$
T_{4}=\frac{P-V X_{1}}{V} .
$$

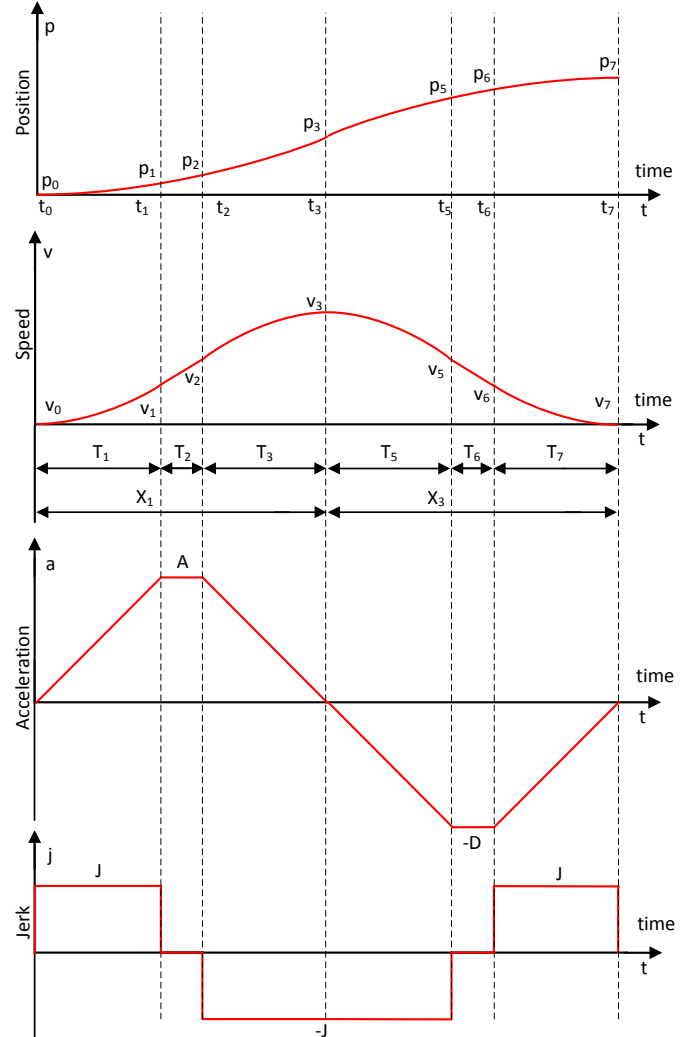

Figure 2. Example of reference trajectory for CASE II.

From (6), (7), and (8), we can find $T_{1}, \ldots, T_{7}$.

\subsection{Reference Trajectory for CASE II}

We next consider the case in which the reference acceleration reaches its maximum value $A$ in the interval $t_{1} \leq t \leq t_{2}$ while the reference speed does not reaches its maximum value. This case corresponds to the case in which the traveling distance is not sufficiently long and thus the position reaches its final value without allowing the speed to reach its maximum value. A typical example of reference trajectory for CASE II is shown in Figure 2. As shown in Figure 2, the reference speed does not reach its maximum value $V$ and thus we can assume that $T_{4}=0$ in this case.

Since the reference acceleration reaches its maximum value and the peak speed of the reference trajectory is lower than the maximum speed, we have the following condition:

$$
P=v_{p}\left(\frac{1}{2} X_{1}+\frac{1}{2} X_{3}\right)=v_{p}\left(2 T_{1}+T_{2}\right),
$$

where

$$
v_{p}=A\left(\frac{1}{2} T_{1}+T_{2}+\frac{1}{2} T_{3}\right)=A\left(2 T_{1}+T_{2}\right) .
$$




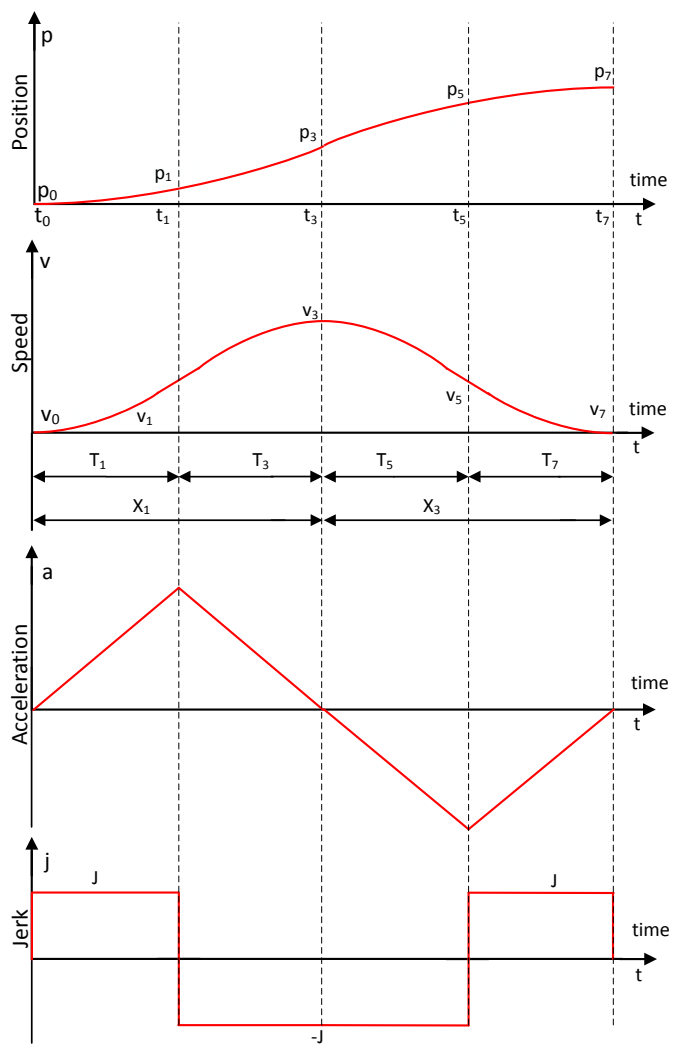

Figure 3. Example of reference trajectory for CASE III.

Due to the fact that the reference acceleration reaches its maximum value at the instance $t=t_{1}$, we have

$$
T_{1}=\frac{A}{J}
$$

By solving the equations $(9)$ and $(10)$, we have the following solution:

$$
T_{2}=\frac{-3 T_{1} \pm \sqrt{T_{1}^{2}+4 P / A}}{2},
$$

where we considers that $T_{2}$ is not negative. Based on (10) and 12), we can find $T_{1}, \ldots, T_{7}$ in this case.

\subsection{Reference Trajectory for CASE III}

We then consider the case in which both the reference speed and reference acceleration do not reach their maximum value during the traveling. This case corresponds to the case in which the traveling distance is too short to allow the reference speed and reference acceleration to reach their maximum value. A typical example of reference trajectory for CASE III is shown in Figure 3 As shown in Figure 3 both of the reference speed
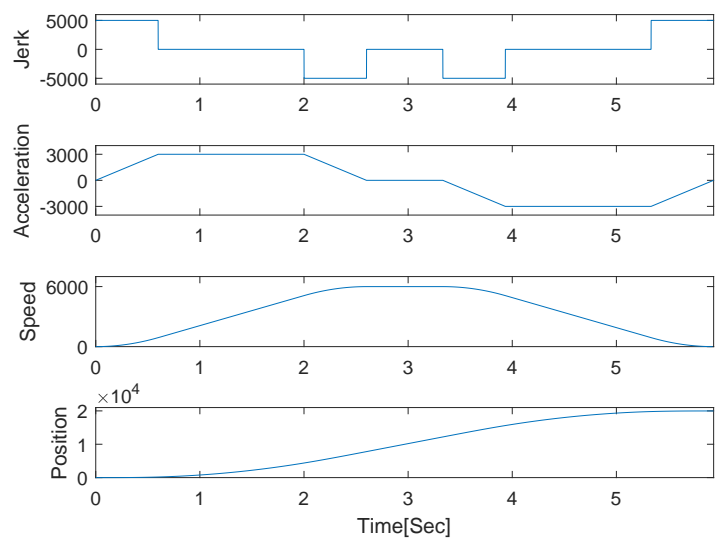

Figure 4. Simulation result for CASE I.
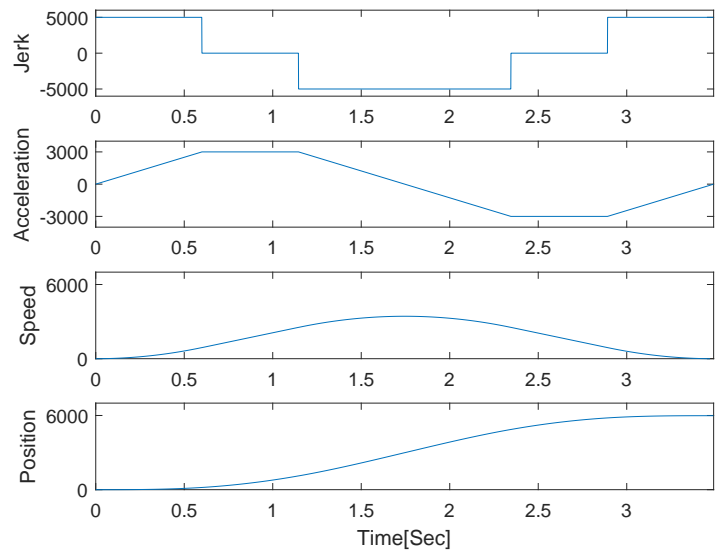

Figure 5. Simulation result for CASE II.

and reference acceleration do not reach its maximum values and thus we can assume that $T_{4}=0$ and $T_{2}=T_{6}=0$ in this case.

For brevity, we denote the peak acceleration by $A_{p}$. Further we express the peak acceleration as

$$
A_{p}=\alpha A,
$$

where $0<\alpha<1$. Then we can obtain

$$
T_{1}=T_{3}=T_{5}=T_{7}=\frac{A_{p}}{J} .
$$

The peak speed is given by

$$
v_{p}=A_{p}\left(\frac{1}{2} T_{1}+\frac{1}{2} T_{3}\right)=\alpha A T_{1} .
$$



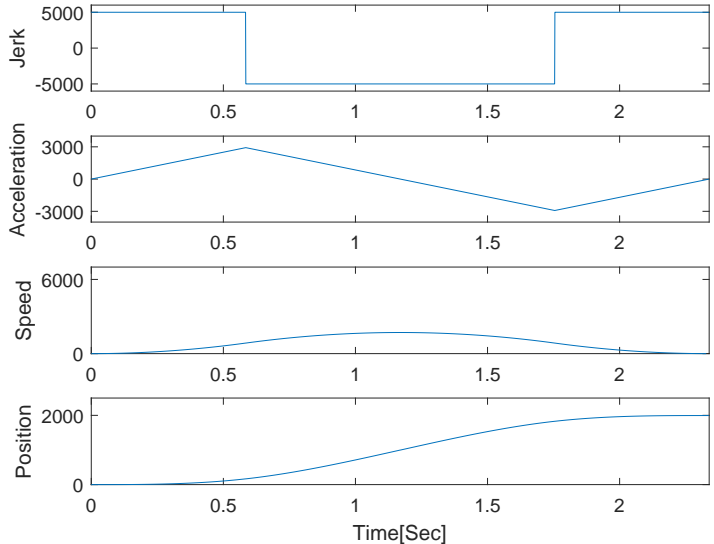

Figure 6. Simulation result for CASE III.

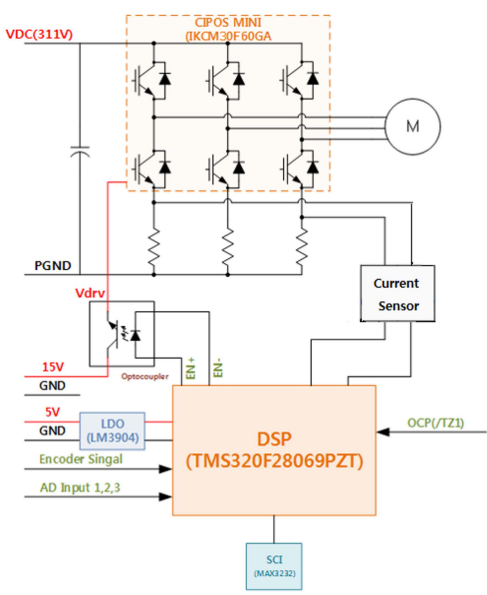

Figure 7. Configuration of the servo motor drive.

From the condition that $P=L$, we have

$$
P=v_{p}\left(\frac{1}{2} X_{1}+\frac{1}{2} X_{3}\right)=2 \alpha^{2} A T_{1}^{2},
$$

which leads us to

$$
A_{p}=\alpha A=\sqrt{\frac{P}{2 A T_{1}^{2}}} .
$$

From (13) and (14), we can have

$$
T_{1}=T_{3}=T_{5}=T_{7}=\left(\frac{P}{2 A J^{2}}\right)^{\frac{1}{4}}
$$

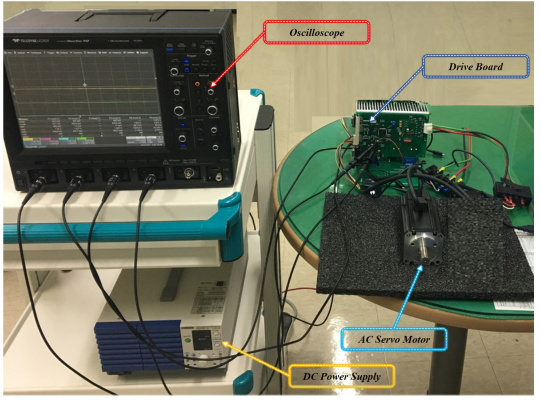

Figure 8. Overall experimental setup.

\section{Simulation}

In this section, we provide simulation results of the jerk-limited time-optimal trajectory generation algorithm. The parameters for the simulation are summarized as follows.

- Maximum jerk: 5,000 degree/ $/ \mathrm{s}^{2}$;

- Maximum acceleration: 3,000 degree $/ \mathrm{s}^{2}$;

- Maximum deceleration: 3,000 degree/ $/ \mathrm{s}^{2}$;

- Maximum speed: 6,000 degree/s;

Figure 4 shows the reference trajectory for CASE I. The traveling distance $P$ is given as $P=20,000$ degree. In this case, the reference jerk, acceleration, and speed values reach their maximum values.

Figure 5 shows the reference trajectory for CASE II.The traveling distance $P$ is given as $P=6,000$ degree. In this case, the reference jerk and acceleration values reach their maximum values while the reference speed does not. The peak speed during the traveling can be found based on (10).

Figure 6 shows the reference trajectory for CASE III. The traveling distance $P$ is given as $P=2,000$ degree. In this case, the peak acceleration needs to be adjusted by (14). From Figure 6, we can check that the reference acceleration is less than its maximum value.

\section{Experiment}

In this section, we provide experimental result of the jerklimited time-optimal trajectory generation algorithm. We first briefly introduce the experimental setup and then provide the experimental results. 


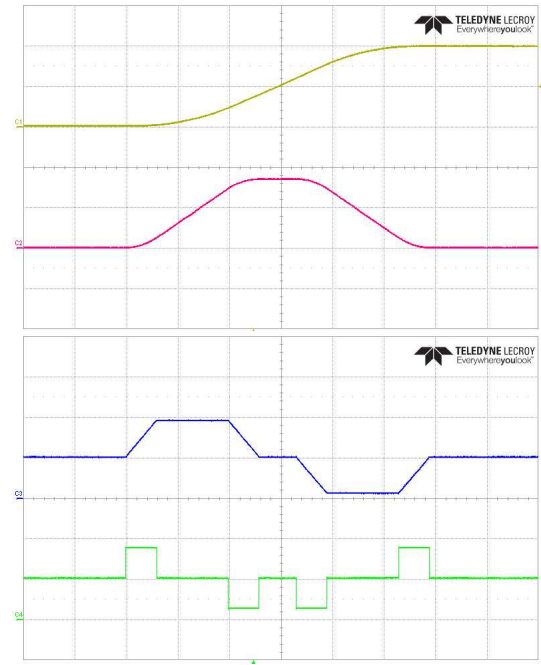

Figure 9. Experimental result for CASE I (yellow: position, red: speed, blue: acceleration, green: jerk).

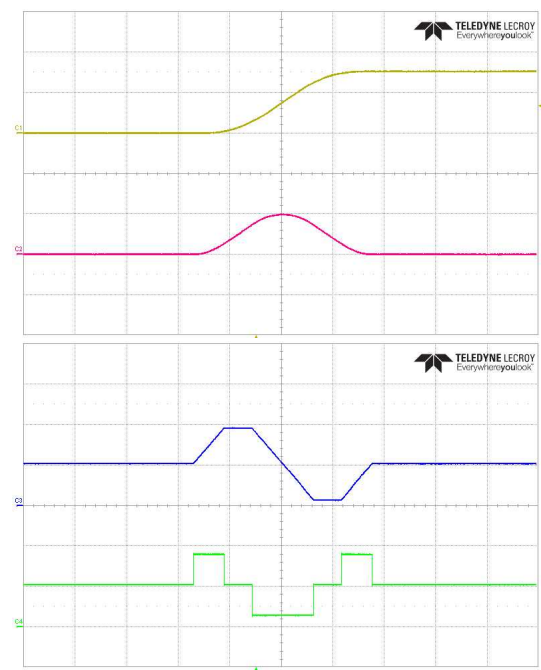

Figure 10. Experimental result for CASE II (yellow: position, red: speed, blue: acceleration, green: jerk).

\subsection{Experimental Setup}

For the experiment, we use a commercial $400 \mathrm{~W}$ class servo motor. We further design and manufacture a servo motor drive based on a relatively low-cost digital signal processor. The overall configuration of the motor drive is shown in Figure 7 A vector control based position controller was implemented in the motor drive [2, 7]. The overall experimental setup is shown in Figure 8 .

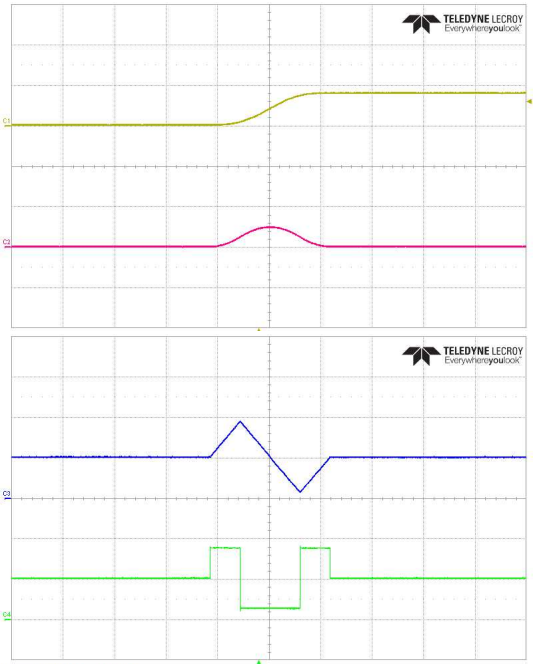

Figure 11. Experimental result for CASE III (yellow: position, ted: speed, blue: acceleration, green: jerk).

\subsection{Experimental Result}

The parameters for the simplified trajectory generation algorithm in the experiment are the same as those in the previous simulation. Figure 9, 10, and 11 show the reference position, speed, acceleration, and jerk trajectories for CASE I, II, and III. As shown in Figure 9 the reference speed and acceleration reach their maximum values during the traveling. On the other hand, the reference speed does not reach its maximum values in the jerk-limited time-optimal trajectories shown in Figure 10 We can see that both of the reference speed and acceleration do not reach their maximum values in CASE III as shown in Figure 11 .

\section{Conclusion}

We implemented a jerk-limited time-optimal trajectory generation algorithm assuming zero initial and final speeds. Considering the computational load imposed by the existing generation algorithm, we provided a simplified version of the algorithm by additionally assuming that the speed values at the initial and final instances are zero. This additional assumption allows us to reduce the computational load of the trajectory generation algorithm. We provided simulation and experimental results to show the validity of the simplified algorithm.

\section{Conflict of Interest}

No potential conflict of interest relevant to this article was reported. 


\section{Acknowledgement}

This work was conducted within the project "Free Piston Engine Linear Generator for CHP” (No. EO170024) at the Korea Institute of Industrial Technology.

\section{References}

[1] M. W. Spong, S. Hutchinson, and M. Vidyasagar, Robot Modeling and Control. New York: John Wiley \& Sons, 2006.

[2] S. K. Sul, Control of Electric Machine Drive Systems. New York: John Wiley \& Sons, 2011.

[3] K. Erkorkmaz and Y. Altintas, "High speed CNC system design. Part I: Jerk limited trajectory generation and quantic spline interpolation," International Journal of Machine Tools and Manufacture, vol. 41, no. 9, pp. 1323-1345, 2001. https://doi.org/10.1016/S0890-6955(01)00002-5

[4] S. Y. Jeong, Y. J. Choi, P. Park, and S. G. Choi, "Jerk limited velocity profile generation for high speed industrial robot trajectories," IFAC Proceedings Volumes, vol. 38, no. 1, pp. 595-600, 2005. https://doi.org/10.3182/200507036-CZ-1902.01369

[5] G. Lee, Y. J. Choi, and J. H. Kim, “Convolution-based desired trajectory generation method considering system specifications," Journal of Institute of Control, Robotics and Systems, vol. 16, no. 10, pp. 997-1005, 2010. http: //doi.org/10.5302/J.ICROS.2010.16.10.997

[6] G. Lee, D. Kim, and Y. Choi, "Faster and smoother trajectory generation considering physical system limits under discontinuously assigned target angles," in Proceedings of 2012 International Conference on Mechatronics and Automation (ICMA), Chengdu, China, 2012, pp. 1196-1201. http://doi.org/10.1109/ICMA.2012.6283421

[7] K. H. Nam, AC Motor Control and Electrical Vehicle Applications. Boca Raton, FL: CRC Press, 2010.

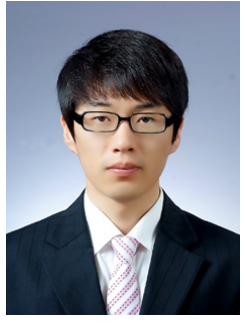

Byeong-Ju Park received the B.S. and M.S. degrees in electrical engineering, Mokpo National University, Jeonnam, Korea, in 2011 and 2013, respectively. He is currently working toward the Ph.D. degree in electrical engineering at Mokpo National University. He is interested in motor control and smart grid design.

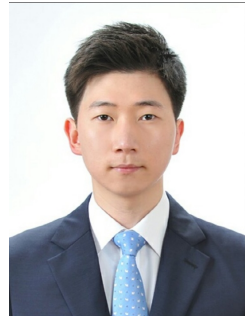

Hong-Jun Lee received the B.S. degree in electronics engineering from Hankuk University of Foreign Studies, Yongin, Korea, in 2016. He is currently working toward the M.S. degree in mechanical engineering at Gwangju Institute of Science and Technology, Gwangju, Korea. He is interested in control of power electronics devices.

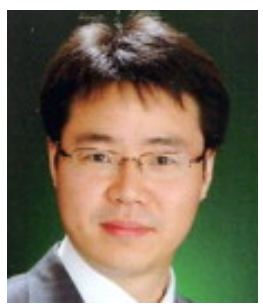

Kwang-Kyo Oh received the B.S. degree in mineral and petroleum engineering and the M.S. degree in electrical and computer engineering from Seoul National University, Seoul, Korea, in 1998 and 2001, and the Ph.D. degree in mechatronics from Gwangju Institute of Science and Technology, Gwangju, Korea, in 2013. He is with Korea Institute of Industrial Technology, Gwangju, Korea. He is interested in control of power electronics devices.

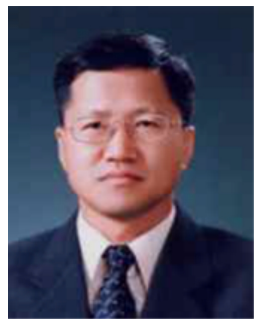

Chae-Joo Moon received the B.S., M.S., and Ph.D. degrees in Electrical Engineering from Chonnam National University, Gwangju, Korea, in 1981, 1983, and 1994, respectively. He has been with the Department of Electrical Engineering, Mokpo National University, where he is currently a full professor. His research interests include design of wind farm and smart grid. 